\title{
Herlyn-Werner-Wunderlich Syndrome: Presentation and Surgical Management Options for Five Cases
}

\author{
Oluwamuyiwa Bolonduro, MD, MPH, ${ }^{1,2}$ Atinuke Akinpeloye, MD, ${ }^{1,2}$ Omar Abuzeid, BA, ${ }^{3}$ \\ Mohammed Ashraf, MD, ${ }^{1-3}$ and Mostafa I. Abuzeid, MD ${ }^{1-3}$
}

\begin{abstract}
Background: Herlyn-Werner-Wunderlich syndrome is a rare Müllerian-duct anomaly consisting of uterine didelphys, a unilateral obstructed hemivagina, and ipsilateral renal agenesis. This article presents clinical and laparoscopic findings as well as the surgical management of 5 cases with various clinical scenarios. Cases: Case 1: An 11-year-old premenarchal female presented with chronic vaginal discharge and negative cultures. She had a uterus didelphys, a unilateral partial obstructed hemivagina, and ipsilateral renal agenesis. She underwent excision of the hemivagina. Case 2: A 14-year-old female presented with severe dysmenorrhea. As part of the work-up for a two-vessel cord at birth, an ultrasound revealed an absent left kidney. Magnetic resonance imaging suggested uterine didelphys and a left hematometrocolpos. At surgery, the MRI findings were confirmed. She underwent excision of the left hemivagina. Case 3: A 23-year-old asymptomatic female was referred following an incidental finding of a duplicated uterine horn and hematocolpos on ultrasound. She had a uterus didelphys, a unilateral obstructed hemivagina, and ipsilateral renal agenesis. She underwent excision of the hemivagina. Case 4: A 26-year-old female presented with severe dysmenorrhea and chronic pelvic pain. She had a uterus didelphys and a high right-sided obstructed hemivagina, with no hematocolpos, hematometra, hematosalpinx, endometrioma, and ipsilateral renal agenesis. She underwent a hemihysterectomy, unilateral salpingectomy, and endometrioma excision. Case 5: A 15-year-old female had a history of severe dysmenorrhea. During laparoscopy for an acute abdomen, she was noted to have uterus didelphys. Further evaluation revealed a hypoplastic cervix and a high right-sided obstructed hemivagina, with minimal hematocolpos, hematometra and ipsilateral renal agenesis. She underwent a hemihysterectomy. Results: The patients recovered well and, in most cases, had complete resolution of their symptoms. In Case 4, the patient's symptoms were greatly reduced. Conclusions: In patients with obstructed hemivaginas that are diagnosed early, resection of the vaginal septum is a convenient and effective treatment modality. Hemihysterectomy should be considered in patients with cervical hypoplasia/ aplasia and high position of obstructed hemivaginas, and in patients with severe endometriosis, tubal damage, and extensive pelvic adhesions. (J GYNECOL SURG 31:46)
\end{abstract}

\section{Introduction}

$\mathbf{H}$ ERLYN-WERNER-WUNDERLICH syndrome is a rare Müllerian-duct anomaly. Its precise etiology and pathogenesis are still unknown. The syndrome has been considered to result from abnormal development of the Wolffian and Müllerian ducts. ${ }^{1}$ The Müllerian ducts differentiate into the Fallopian tubes, uterus, cervix and upper vagina. Defects of Müllerian ducts differentiation-such as agenesis, hypoplasia, and defects of vertical and lateral fusion-will lead to uterine and vaginal anomalies. ${ }^{2}$ These patients may present with uterine didelphys, obstructed hemivaginas and ipsilateral renal agenesis. ${ }^{3}$ Because the genital system arises from a common embryonic mesoderm, maldevelopment of the paramesonephric ducts also may interfere with embryogenesis of the kidneys and ureters. Therefore, the evolution of uterus didelphys with a blind hemivagina and ipsilateral renal agenesis simultaneously involves the mesonephric and paramesonephric ducts. ${ }^{4}$

\footnotetext{
${ }^{1}$ Division of Reproductive Endocrinology and Infertility, Department of Obstetrics and Gynecology, Hurley Medical Center, Flint, MI. ${ }^{2}$ Michigan State University, College of Human Medicine, Flint Campus, Flint, MI.

${ }^{3}$ IVF Michigan, PC, Rochester Hills, MI.
} 
The triad of uterus didelphys, obstructed hemivagina, and ipsilateral renal anomaly was first reported in $1950 .^{5}$ The incidence of uterus didelphys associated with Herlyn-Werner-Wunderlich syndrome is between 1:2000 and 1:28000. ${ }^{6}$ It is accompanied by an absent kidney in $43 \%$ of cases. ${ }^{6}$ The condition usually is not detected until puberty, after menstrual blood accumulates in the obstructed side. Increasing menstrual effluent then distends the vagina, uterus, and the Fallopian tube, and eventually spills into the pelvic cavity. ${ }^{7}$ This syndrome's rarity and variable clinical features may contribute to a diagnostic delay for years after menarche. ${ }^{4}$

The initial clinical diagnosis is incorrect in a significant portion of cases because of the rarity of this anomaly. Because of the association with menstrual disorders, endometriosis, and, in later years, with primary infertility, an accurate diagnosis is important. ${ }^{1}$

This report presents a case series of 5 patients with various clinical courses. The clinical and laparoscopic findings are also discussed as well as the surgical management in the various clinical scenarios.

\section{Cases}

Five cases of Herlyn-Werner-Wunderlich syndrome were treated at the Hurley Medical Center in Flint, MI, and IVF Michigan, Rochester Hills, MI, between January 2012, and March 2014.

\section{Case 1}

An 11-year old, premenarcheal female was referred to the current authors by her pediatrician for evaluation of chronic leukorrhea with negative cultures. The patient's general physical examination was unremarkable. A transabdominal sonogram result was suspicious for two uterine cavities. Magnetic resonance imaging (MRI) revealed symmetrical uterine didelphys, two cervices, a possible right hemivagina, and an absent right kidney. On laparoscopy, two parallel uterine horns and normal adnexae bilaterally were noted. Diagnostic vaginoscopy revealed no foreign bodies, a normal left cervix, and a thin right hemivagina with a micro-perforation through which leukorrhea seeped. The septum was incised and a significant amount of whitish discharge was noted. Exploration of this opening revealed a distinct cervix. The septum was subsequently resected. The patient's postoperative course was uneventful and her symptoms subsided.

\section{Case 2}

A 14-year-old female, nulligravida, presented with complaints of severe dysmenorrhea and irregular menses that were attributed to anovulatory cycles. As part of a work-up for a two vessel cord at birth, an ultrasound scan was performed. It showed an absent left kidney. Transabdominal ultrasound results were suggestive of a bicornuate uterus, while a subsequent MRI revealed uterine didelphys, left hematocolpos, and left hematometra. After due counseling of the patient and her family, she was placed on a gonadotropin-releasing hormone agonist for symptom management and a diagnostic laparoscopy and examination under anesthesia were scheduled.

In the operating room, a pelvic examination revealed a distended mass that was palpated on the left side of the patient's vagina. A uterus didelphys was confirmed by laparoscopy.
Other findings included an enlarged left uterus suggestive of hematometra, a large mass consistent with left hematocolpos (Fig. 1) and minimal left peritubal adhesions. There was a normal-appearing right uterus and Fallopian tube. Both ovaries were normal. Left salpingolyis was performed to restore the normal anatomy. Widespread stage II endometrosis was noted, which was coagulated to the extent possible. The palpable left paravaginal bulge was incised with drainage of copious amounts of old menstrual effluent. The septum was then excised and the edges of the vaginal mucosa were approximated using 2-0 vicryl in an interrupted manner. The patient did well post-operatively. Her pelvic pain has since resolved.

\section{Case 3}

A 23-year-old female, nulligravida, was referred to the current authors following the incidental finding of a duplicated uterine horn and right hematocolpos, noted on ultrasound. The ultrasound was ordered because a palpable right paravaginal mass was noted during her annual physical examination. The patient's menarche was at age 13. She reported a history of irregular menses, which had been managed for the past 8 years by oral contraceptive pills. She denied dysmenorrhea or pelvic pain. Her physical examination was remarkable for a vaginal bulge on the right side. The left cervix appeared to be normal. An MRI revealed uterine didelphys, with an enlarged right horn suggesting hematometra, right hematocolpos, and right renal agenesis.

After due counseling, the patient was scheduled for a diagnostic laparoscopy and examination under anesthesia with possible vaginal septoplasty. Examination under anesthesia revealed a right-sided obstructed hemivagina. Laparoscopy confirmed uterine didelphys (Fig. 2) and a small bulge inferior to the right uterosacral ligament was noted (Fig. 3). The septum was excised and the edges of the vaginal mucosa were approximated using 2-0 vicryl in an interrupted manner. The patient did well postoperatively.

\section{Case 4}

A 26-year-old female, nulligravida, sought treatment for a long history of severe dysmenorrhea, occasionally requiring hospitalization for pain management. Her history was significant for menarche at age 10, regular menses lasting 7 days with a normal flow, a bicornuate uterus, and a unilateral left kidney. Her a pelvic examination was significant for a single

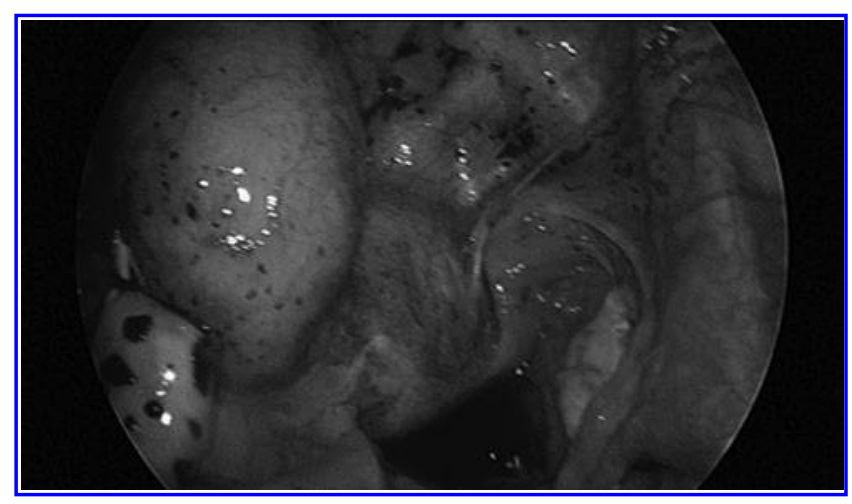

FIG. 1. Distended left uterus and enlarged hematocolpos (case 2). 


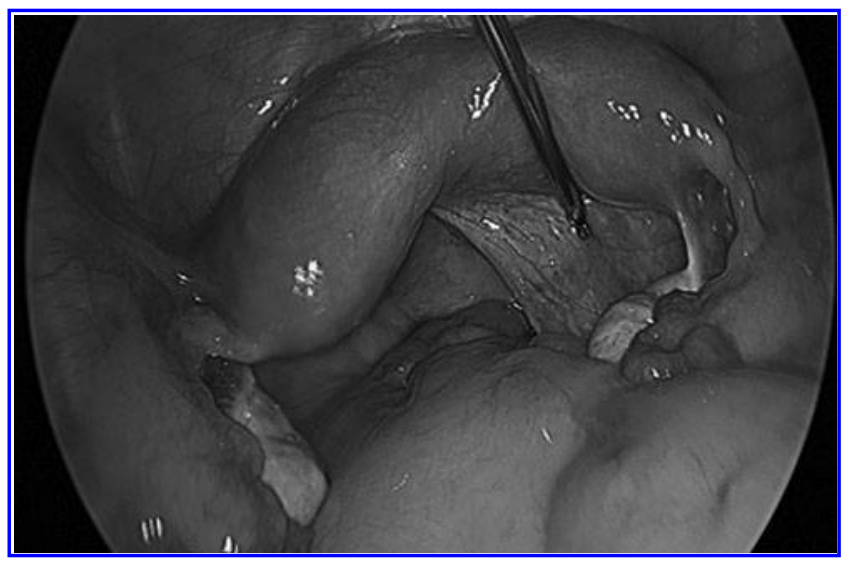

FIG. 2. Uterus didelphys (case 3).

cervix and vagina. No bulges, septa, or obstructions were noted. A transvaginal ultrasound result was suspicious for uterus didelphys, right hematometra, right hematosalpinx, and possible endometrioma. A confirmatory MRI showed uterine didelphys with right-sided hematometra, hematosalpinx, and a possible hypoplastic/aplastic right cervix, and no evidence of hematocolpos. The above findings were discussed with the patient and she requested definitive treatment.

Laparoscopy confirmed the imaging findings (Figs. 4 and 5). Considering the laparoscopy findings, the patient's history of multiple admissions for pain management, and her desire for definite resolution of her symptoms, a DaVinci robotic assisted laparoscopic right hemihysterectomy, excision of the right hemivagina, right salpingectomy, excision of right endometrioma, and coagulation of endometriotic implants were performed. The right uterus and Fallopian tube were removed

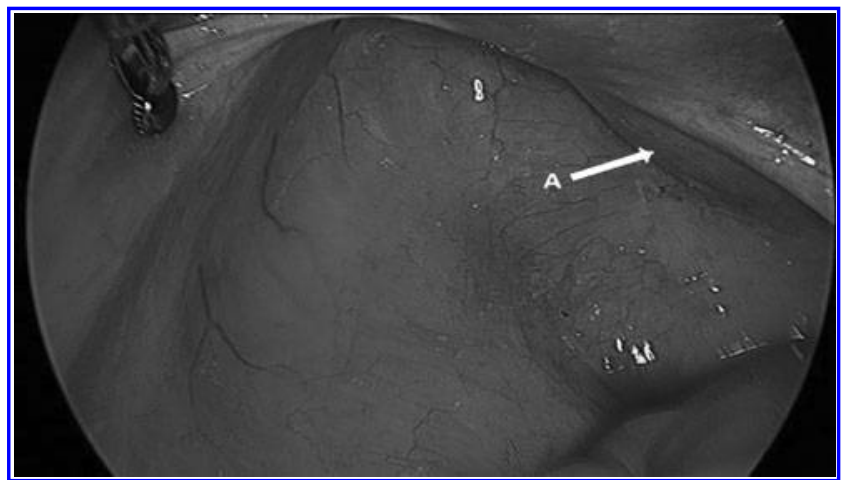

FIG. 3. Distended right hemivagina inferior to uretosacral ligament (case 3). See arrow A.

through the vaginal vault which was then reapproximated with a few interrupted sutures of 2-0 vicryl. This patient recovered well, and her postoperative course was uncomplicated. Her condition was improved greatly since the surgery.

\section{Case 5}

A 15-year-old female with a history of severe dysmenorrhea presented to the emergency room with complaints of severe, bilateral lower-quadrant pain, chills, nausea and vomiting. She reported a history of cyclic abdominal pain since her menarche, occasionally requiring hospitalization for pain management. She was on the third day of her menses. The patient reported menarche at age 13 with regular menstrual cycles. She was not sexually active.

Computed tomography results and clinical findings were suspicious for appendicitis. She underwent emergent laparoscopy, which revealed a normal appendix, a large amount

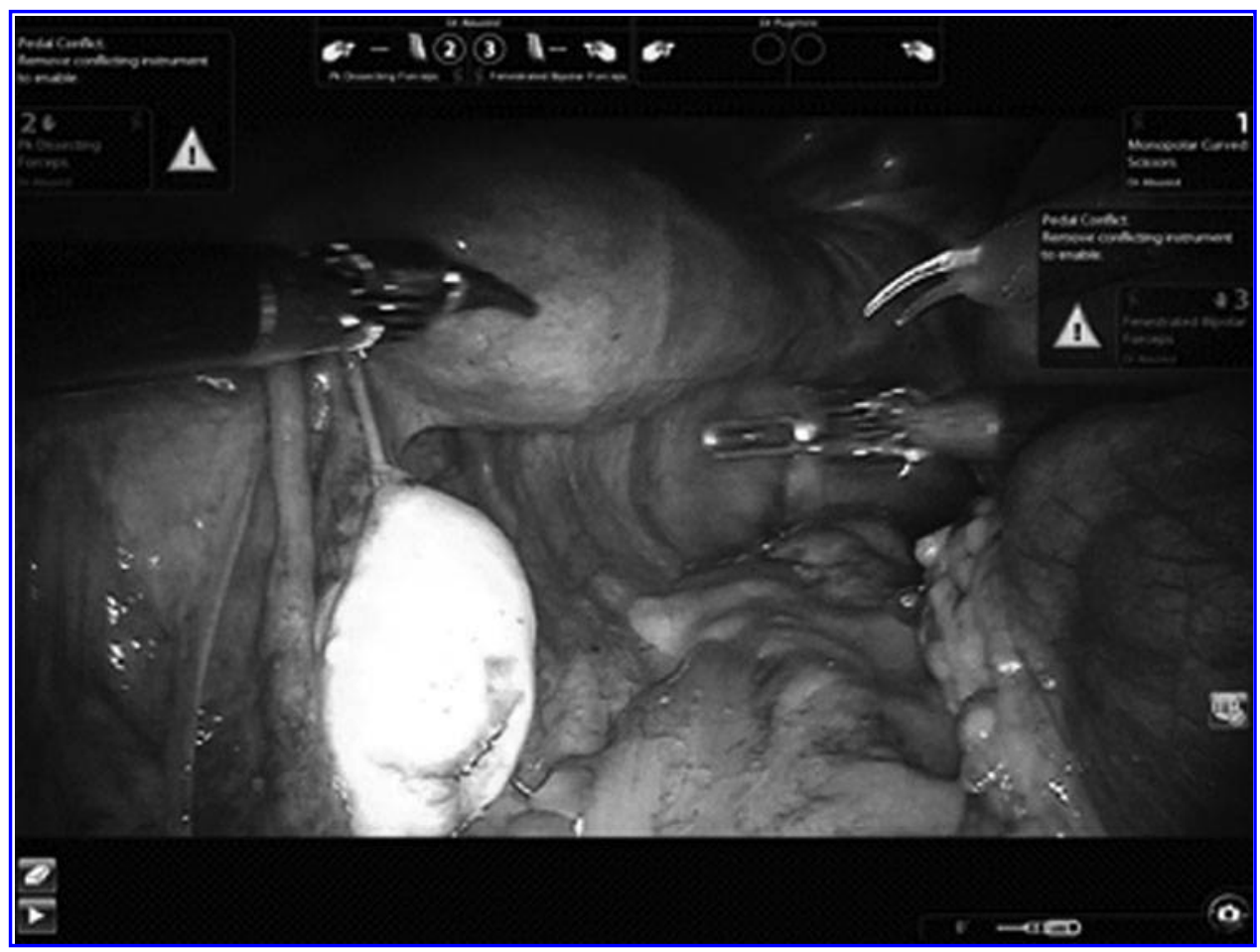

FIG. 4. Normal left uterine horn, Fallopian tube, and ovary (case 4). 


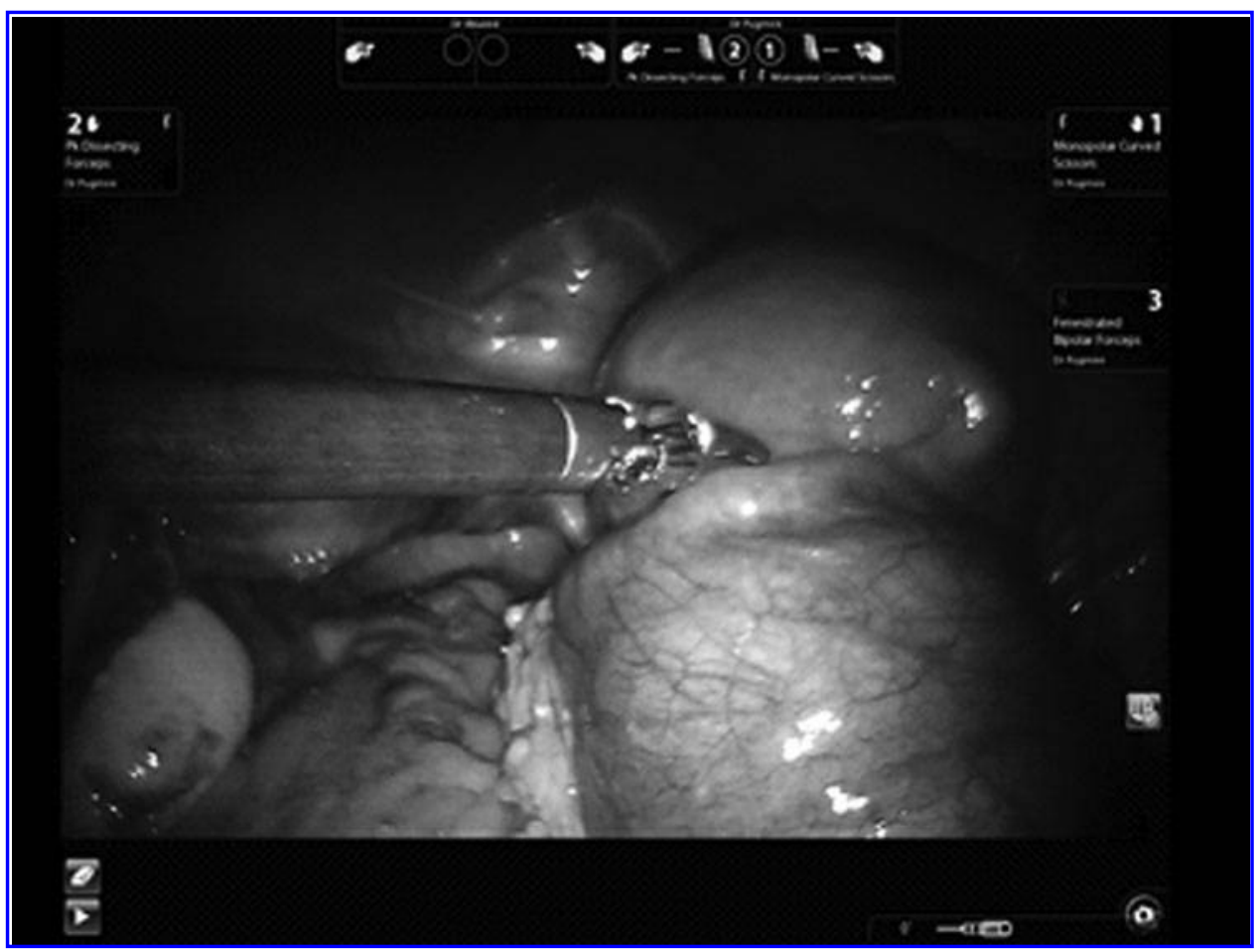

FIG. 5. Distended right uterine horn, right hematosalpinx, and endometrioma (case 4).

of old blood in the pelvis, and uterine didelphys. The surgery was terminated and the patient was subsequently referred to the current authors for further management. An MRI performed afterward was significant for asymmetric uterine didelphys suggestive of right-sided hematometra, minimal hematocolpos, suspected hypoplastic right cervix, and ipsilateral renal agenesis. The left cervix appeared to be normal.

The patient and her family were counseled about the MRI findings. Management options were discussed, including a vaginal septum resection; its complications, which could include the need for a two-stage vaginoplasty if the septum were thick; or, in case of of an incomplete resection, the risks of stenosis, infection, bleeding, anatomical distortion, and the possible need for a hemihysterectomy if cervical hypoplasia/aplasia were to be confirmed.

Examination under anesthesia revealed a single cervix and vagina. No bulge, septum, or obstruction were noted in that area. During operative laparoscopy, an asymmetric uterus didelphys, with the right uterus higher in position, compared to the left uterus, and minimal right hematocolpos were observed (Fig. 6). Widespread endometriosis seen in the pelvis was coagulated as appropriate. The right uterine horn was followed down to the vagina and a transverse incision was made in the posterior wall of what felt like a small hematocolpos. A small amount of dark red blood was suctioned out. Further examination revealed a poorly formed hypoplastic cervix.

Because of concerns about worsening hematometra, endometriosis, chronic pelvic pain, and eventual infertility, a decision was made to proceed with a DaVinci, roboticassisted, laparoscopic right hemihysterectomy, right salpingectomy, and excision of the right hemivaginal septum. The right uterus and Fallopian tube were removed through the vaginal vault, which was then approximated with a few interrupted sutures using 2-0 vicryl. The patient recovered well, and her postoperative course was significant for symptom resolution.

\section{Results}

As noted in each case, the patients recovered well and, in most cases, had complete resolution of their symptoms. In Case 4 , the patient's symptoms were greatly reduced.

\section{Discussion}

Since the first report of uterus didelphys, obstructed hemivagina, and ipsilateral renal anomaly in 1950, >200

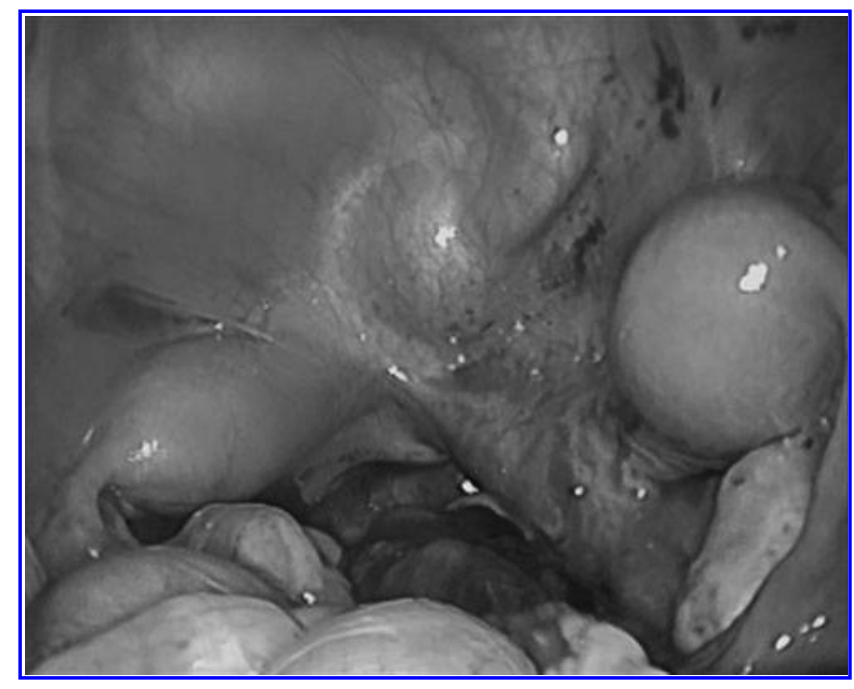

FIG. 6. Distended right horn, endometriosis, and hematoperitoneum (case 5). 
such cases have been reported in the literature. ${ }^{5}$ This report includes 4 cases with right-sided obstructions and 1 case with a left-sided obstruction. This is in accordance with previously reported literature stating a higher incidence of right-sided obstruction, compared to left-sided obstruction. ${ }^{5}$ The typical presentation of a patient with HerlynWerner-Wunderlich syndrome is an adolescent who, following menarche, complains of progressing dysmenorrhea and has a vaginal mass noted on pelvic examination. ${ }^{1,8}$ Because the above triad is not a common cause of pelvic pain and menstrual discomfort in young women, appropriate imaging and, thus, diagnosis is delayed. Any adolescent presenting with these symptoms should have an ultrasound to assess her uterine anatomy. ${ }^{5}$

Case 1 differed from this typical scenario because she presented before menarche and had an incompletely obstructed hemivagina (a microperforation in the septum) through which accumulated mucocolpos seeped continually. Leukorrhea has previously been reported in association with obstructed hemivagina. Hansen and De Witt reported a 5-year-old-girl, with a small perforation in the obstructed hemivagina, who presented with recurrent urinary-tract infections (UTIs) and chronic vaginal discharge. ${ }^{8}$ The researchers speculated that the patient's UTIs could have been related to the obstruction from the mucocolpos. Obstruction in urinary flow may result in stasis, thus increasing the risk for bacteria proliferation and recurrent UTIs. ${ }^{8}$ Evaluation of a patient with a uterine anomaly primarily involves a taking history and conducting a physical examination, combined with appropriate imaging. ${ }^{8}$

In case $1-\mathrm{a}$ virginal female with recurrent vaginal discharge and MRI results that were suggestive of mucocolposan examination under anesthesia with diagnostic vaginoscopy helped confirm the anomaly prior to surgical resection.

Case 2 represents the classic presentation of HerlynWerner-Wunderlich syndrome. This patient presented 2 years after menarche with pelvic pain and a palpable vaginal mass. Several studies have reported patients who presented similarly to case 2. Beekhuis and Hage described 2 patients who presented with pelvic pain, each with a vaginal mass. ${ }^{9}$ Following the diagnosis of Herlyn-Werner-Wunderlich syndrome, the patients underwent septum resection with symptom resolution.

For patients, with the classic presentation (a bulging obstructed hemivagina), excision of the hemivaginal septum and draining of its contents is the treatment of choice. ${ }^{1,8-10}$ Incision-only should be avoided because of concerns about spontaneous closure, which might result in recurrence of symptoms. $^{11,12}$

Case 3 exemplifies the variability of presentation of patients with Herlyn-Werner-Wunderlich syndrome. Although this patient had a hematocolpos, which was presumed to be responsible for the pelvic pain, ${ }^{13}$ she did not have dysmenorrhea or associated pelvic pain. She was also noted to have minimal endometriosis, as opposed to cases 2, 4, and 5. It has been shown in a few studies that accurate diagnosis of Müllerian anomalies can be delayed by several weeks to years from initial presentation. ${ }^{5}$ The mean age of diagnosis is 17.4 years' old. ${ }^{13}$

One of the patients in this series (case 4) had been diagnosed earlier in her life with a bicornuate uterus. Her pain was explained as arising from her endometriosis, which is known to be associated with Müllerian anomalies. ${ }^{14}$ Her eventual diagnosis, 16 years after menarche, was late as suggested by the findings of a large hematometra, hematosalpinx, endometrioma, and pelvic adhesions.

Early diagnosis in case 5 was a result of a laparoscopy for suspected appendicitis. Although this patient had presented to the hospital on several occasions for severe pelvic pain, she had never undergone a sonogram to establish uterine anatomy. Early diagnosis requires a high index of suspicion and can be achieved by taking a careful history, gynecologic examination, and appropriate imaging tests. If a Müllerian anomaly is suspected, appropriate follow-up should include an MRI, preferably at a center with expertise in interpretation of Müllerian anomalies. ${ }^{1,5}$

Because of poorly formed cervices and minimal hematocolpos, neither case 4 nor case 5 had a palpable bulge/mass on pelvic examination, hence, resulting in a management dilemma. The presence of a hypoplastic cervix and marked asymmetry of the two uteri and cervices in cases 4 and 5 necessitated hemihysterectomy and septum resection. Saleh and Badawy described a patient with uterine didelphys and a unilateral obstruction caused by cervical atresia, ${ }^{15}$ Donnez et al. described a case of recurrent hematometra secondary to the absence of the recovery of normal uterine contractility after septum resection. ${ }^{11}$ Zuwarin et al. described a patient with ureteral obstruction and a compromised transplanted kidney caused by hematometra and hematosalpinx, and a second case of an ascending infection through a small opening in the vaginal septum that led to an abscess. ${ }^{16}$ Haddad et al. reported on a 42-patient case series, 5 of whom had severe adnexal complications including adhesions. ${ }^{12}$ To reduce the risk of worsening symptoms in the above cases, hemihysterectomy and septum resection were performed. ${ }^{11,12,15,16}$

The mere presence of endometriosis (cases 2 and 3) and hematosalpinx should not be considered indications for hemihysterectomy. In patients who present with palpable hematocolpos, hemihysterectomy can be avoided in favor of excision of the hemivagina, conservative management of endometriosis, salpingectomy, and lysis of adhesions. ${ }^{1,6,9}$ A hemihysterectomy should only be considered in cases of endometriosis that have resulted in severe adnexal involvement and extensive pelvic adhesions, especially if the patients' symptoms are severe and unresponsive to conservative management. ${ }^{15,17}$

Laparoscopy has been advocated as the "gold standard" for complete evaluation of congenital anomalies of the female reproductive tract. This is particularly so for nonmidline structural anomalies, as shown by a cohort of 22 patients-comparing laparoscopy and MRI as diagnostic tools-in whom only $53 \%$ of Müllerian anomalies diagnosed by laparoscopy were noted on MRI. ${ }^{5,16}$ To the current authors' knowledge, this is the first report of robotic-assisted hemihysterectomy and septum dissection. The current authors propose a role for robotic surgery in complex cases because of improved visualization, and meticulous dissection and dexterity. In addition, robotic surgery is associated with less blood loss and easier reapproximation of defects. ${ }^{18}$

\section{Conclusions}

Herlyn-Werner-Wunderlich syndrome should be considered among the differential diagnoses in young females with renal anomalies presenting with severe dysmenorrhea, pelvic 
pain, and pelvic masses. ${ }^{1}$ In such patients, early diagnosis and treatment is essential to avoid obstruction of menstrual effluent, which could lead to complications, such as chronic pelvic pain, ureteral obstruction, retrograde menstruation, endometriosis, and eventual infertility. ${ }^{16,19}$

Early resection of the vaginal septum is an effective modality for managing classic cases with hematocolpos. Hemihysterectomy is a management option for patients with cervical hypoplasia/aplasia, high position of obstructed hemivagina with no hematocolpos, and in patients with severe endometriosis, adnexal damage, and extensive pelvic adhesions. ${ }^{15,17}$ Hemihysterectomy should also be considered in patients with uterus didelphys and a hemivagina that fails to regain normal uterine myometrial function (recurrent hematometra) after complete transvaginal septum resection. ${ }^{11}$

DaVinci robotic surgery can play a role in complex cases because of improved visualization, meticulous dissection and dexterity.

\section{Disclosure Statement}

No competing financial conflicts of interest exist.

\section{References}

1. Mandava A, Prabhakar RR, Smitha S. OHVIRA Syndrome (obstructed hemivagina and ipsilateral renal anomaly) with uterus didelphys: An unusual presentation. J Pediatr Adolesc Gynecol 2012;25:23.

2. Wu TH, Wu TT, Ng YY, et al. Herlyn-Werner-Wunderlich syndrome consisting of uterine didelphys, obstructed hemivagina and ipsilateral renal agenesis in a newborn. Pediatr Neonatol 2012;53:68.

3. Takagi H, Matsunami K, Imai A. A uterovaginal duplication with blind hemivagina and ipsilateral renal agenesis: Review of unusual presentation. J Obstet Gynaecol 2010; 30:350.

4. Varras M, Akrivis CH, Karadaglis S, Tsoukalos G, Plis Ch, Ladopoulos I. Uterus didelphys with blind hemivagina and ipsilateral renal agenesis complicated by pyocolpos and presenting as acute abdomen 11 years after menarche: Presentation of a rare case with a review of the literature. Clin Exper Obstet Gynecol 2008;35:156.

5. Kimble RM, Khoo SK, Baartz D, Kimble RM. The obstructed hemivagina, ipsilateral renal anomaly, uterus didelphys triad. Aust N Z J Obstet Gynaecol 2009;49:554.

6. Del Vescovo R, Battisti S, Di Paola V, et al. HerlynWerner-Wunderlich syndrome: MRI findings, radiological guide (two cases and literature review), and differential diagnosis. BMC Med Imaging 2012;12:4.

7. Lin CC, Chen AC, Chen TY. Double uterus with an obstructed hemivagina and ipsilateral renal agenesis: Report of 5 cases and a review of the literature. J Formos Med Assoc 1991;90:195.
8. Hansen K, DeWitt J. Premenarchal, recurrent vaginal discharge associated with an incomplete obstructing longitudinal vaginal septum. J Pediatr Adolesc Gynecol 2005; $18: 423$.

9. Beekhuis JR, Hage JC. The double uterus associated with an obstructed hemivagina and ipsilateral renal agenesis. Eur J Obstet Gynaecol Reprod Biol 1983;16:47.

10. Mane SB, Shastri P, Dhende NP, et al. Our 10-year experience of variable Müllerian anomalies and its [sic] management. Pediatr Surg Int 2010;26:795.

11. Donnez O, Jadoul P, Squifflet J, Donnez J. Didelphic uterus and obstructed hemivagina: Recurrent hematometra in spite of appropriate classic surgical treatment. Gynecol Obstet Invest 2007;63:98.

12. Haddad B, Barranger E, Paniel BJ. Blind hemivagina: Long term follow up and reproductive performance in 42 cases. Hum Reprod 1999;14:1962.

13. Tanaka YO, Kurosaki Y, Kobayashi T, et al. Uterus didelphys associated with obstructed hemivagina and and ipsilateral renal agenesis: MR findings in seven cases. Abdom Imaging 1998;23:437.

14. Rackow BW, Arici A. Reproductive performance of women with Müllerian anomalies. Curr Opinion Obstet Gynecol 2007;19:229.

15. Saleh M, Badawy SZ. Unilateral non-communicating cervical atresia in a patient with uterus didelphys and unilateral renal agenesis. J Pediatr Adolesc Gynecol 2010; 23:137.

16. Zuwarin R, Dietrich J, Heard M, Edwards C. Didelphic uterus and obstructed hemivagina with renal agenesis: Case report and review of the literature. J Pediatr Adolesc Gynecol 2004; 17:137.

17. Skondras KG, Moutsouris CC, Vaos GC, Barouchas GC, Demetriou LD. Uterus didelphys with an obstructed hemivagina and ipsilateral renal agenesis: A rare cause of acute abdomen in pubertal girls. J Pediatr Surg 1991; 26:1200.

18. Anderberg M, Bossmar T, Arnbjornsson E, Isaksson J, Persson J. Robotic assisted laparoscopic hemihysterectomy for rare genitourinary malformation. Eur J Pediatr Surg 2010;20:206.

19. Schutt AK, Barrett MR, Trotta BM, Stovall DW. Peri-operative evaluation in Herlyn-Werner-Wunderlich syndrome. Obstet Gyneol 2012;124:948.

Address correspondence to: Oluwamuyiwa Bolonduro MD, MPH

Division of Reproductive Endocrinology and Infertility Department of Obstetrics and Gynecology, Hurley Medical Center,

Two Hurley Plaza, Suite 209 Flint, MI 48503

E-mail: obolond1@hurleymc.com 
This article has been cited by:

1. Pedro Acién, Maribel Acién. 2016. The presentation and management of complex female genital malformations. Human Reproduction Update 22:1, 48-69. [CrossRef] 